\title{
The conundrum of contrast-induced acute kidney injury
}

\author{
Mohammad Faraz Ul Haq ${ }^{1}$, Cindy S. Yip ${ }^{1}$, Pradeep Arora ${ }^{1,2}$ \\ ${ }^{1}$ Department of Medicine, SUNY at Buffalo, NY, USA; ${ }^{2}$ Division of Nephrology at VAMC, Buffalo, NY, USA \\ Contributions: (I) Conception and design: P Arora; (II) Administrative support: None; (III) Provision of study materials or patients: None; \\ (IV) Collection and assembly of data: None; (V) Data analysis and interpretation: None; (VI) Manuscript writing: All authors; (VII) Final approval of \\ manuscript: All authors. \\ Correspondence to: Pradeep Arora, MD. Division of Nephrology at VAMC, Buffalo, Department of Medicine, SUNY at Buffalo, 3495 Bailey Ave., \\ Buffalo, New York 14215, USA. Email: Pradeep.arora@va.gov.
}

\begin{abstract}
More than sixty years have elapsed since contrast induced nephropathy (CIN) was first described in the medical literature. This term has since been extensively explored, with a variety of studies conducted to investigate its incidence and various mechanisms examined to explain its pathophysiology. However, the topic of CIN remains one of controversy with a widely variable and often questionable incidence derived from various studies. The past two decades have seen a surge in reports questioning the existing of CIN altogether and if more harm is actually being caused to patients out of fear of this potential complication. We have attempted to review relevant studies regarding CIN and highlight the key points of its surmised understanding. The review has a higher focus on more recent literature and updates, in order to determine if an accurate estimate can be made on the incidence of CIN. While there was certainly no lack of material available, practically all the studies reviewed were limited by one or more significant drawbacks that limited the reliability of their conclusions regarding CIN. Based on the information reviewed, the strengths and the flaws encountered in other studies can be used to design a randomized control trial that may help in concluding the longstanding debate on this topic. However due to time, financial, and perhaps even ethical constraints such a trial will be difficult to arrange, and so a definitive answer on CI-AKI, and whether it really exist, may continue to elude clinicians.
\end{abstract}

Keywords: Radiocontrast media; acute kidney injury (AKI); outcomes

Submitted Oct 14, 2019. Accepted for publication Dec 16, 2019.

doi: $10.21037 /$ jtd.2019.12.88

View this article at: http://dx.doi.org/10.21037/jtd.2019.12.88

\section{Background}

Medical imaging plays a pivotal role as an important diagnostic and therapeutic tool in the management of patient care in an era of great technological advances. Radiocontrast media are increasingly used to better enhance imaging in diagnostic computed tomography $(\mathrm{CT})$, procedures of interventional radiology and percutaneous coronary intervention (PCI). The use of radiocontrast media, however, is not without side effects. One of the common complications with intravascular radiocontrast media exposure is the development of an acute kidney injury (AKI), first described in the 1950s in a patient with multiple myeloma $(1,2)$. Since then, thousands of research papers related to contrast-induced acute kidney injury (CI-AKI) in both experimental and clinical studies have been published (3-10).

Indeed, CI-AKI is the third most common cause of hospital acquired AKI, representing about $12 \%$ of the cases, surpassed only by renal hypoperfusion (42\%) and postoperative renal injury [18\% (11)]. In most patients, CI-AKI may be transient and reversible in nature, although it has also been reported to be associated with increased long-term mortality in several studies $(9,10,12)$. Recently, several research manuscripts have shown that radiocontrast media use may not be associated with AKI as similar rates of AKI were shown in patients who underwent contrast enhanced CT compared with those who underwent 
Table 1 Proposed pathophysiological mechanisms of contrast-induced acute kidney injury [adapted from reference (25)]

\author{
Medullary vasoconstriction and hypoxia \\ Direct cytotoxicity to renal tubular cells \\ Release of vasoconstrictive mediators: Endothelin, adenosine, angiotensin II, vasopressin \\ Reduction of vasodilatatory mediators: Nitric oxide, prostocyclin \\ Increased oxidative stress \\ Impairment of tubulo-glomerular feedback \\ Increased blood and renal tubular viscosity \\ Impairment of mitochondrial function and mitochondrial membrane potential
}

unenhanced CT imaging (13-15). Thus far, it is not clear if CI-AKI is directly a cause of increased long-term mortality or merely a marker of high-risk patients, such as those with AKI or preexisting chronic kidney disease (CKD). Initial studies of AKI following intravenous (IV) radiocontrast media were limited by the lack of control groups and/ or did not adjust for additional covariates associated with AKI development. More contemporary studies have used propensity score-analyses to minimize selection bias, although without a prospective, randomized controlled trial, bias cannot be further eliminated.

\section{Definition and epidemiology}

There is no uniform definition of CI-AKI and this has hampered comparisons across studies. Traditionally, CIAKI was diagnosed based on two measurements of serum creatinine levels [before and after radiocontrast media exposure (4,16-20)]. The most accepted definition was an absolute $(\geq 0.5 \mathrm{mg} / \mathrm{dL})$ or a relative $25 \%$ increase in baseline serum creatinine concentration, assessed at 48 to 72 hours after a radiological procedure (18). This definition has been shown to correlate well with major adverse events and mortality on follow up studies. Of note, the diagnosis is notably one of exclusion and can only be made if AKI cannot reasonably be attributed to another etiology.

The incidence of CI-AKI has been reported to be $>2 \%$ in the general population, however, the incidence increases to $20 \%$ to $40 \%$ in high-risk patients, for example, in patients with diabetes, congestive heart failure, CKD and older age. Each year, there are approximately 150,000 patients with CI-AKI reported in the world, and at least $1 \%$ requiring dialysis and prolonged hospital stay with increased cost of care and morbidity $(5-10,12,21,22)$. The entity of CI-AKI was coined in association with coronary angiography, despite $90 \%$ of all intravascular radiocontrast media being administered intravenously during CT studies. In a large national study, AKI incidence in patients hospitalized with acute myocardial infarction declined significantly from 2000 to 2008 despite the aging population and rising prevalence of AKI risk factors, where the reported AKI incidence declined from $26.6 \%$ in 2000 to $19.7 \%$ in $2008(\mathrm{P}<0.001)$ amongst patients who were admitted with acute myocardial infarction (23). However, these findings likely reflected increased clinician awareness, better risk stratification, or greater use of AKI prevention efforts during this time period.

Nevertheless, factors associated with more than doubling in the rates of CI-AKI and significant increases in the risk of AKI requiring dialysis include ST-segment elevation myocardial infarction (STEMI), eGFR $<30 \mathrm{~mL} / \mathrm{min} / 1.73 \mathrm{~m}^{2}$, and cardiogenic shock (24). Mehran et al. proposed a CI-AKI risk stratification score, where an increasing score number conferred an exponentially increased CI-AKI risk (16). The variables included in the CIN risk score were: (I) patientrelated characteristics (i.e., age $>75$ years, diabetes mellitus, chronic congestive heart failure, or admission with acute pulmonary edema, hypotension, anemia, and CKD); and (II) procedure-related characteristics (i.e., the use of elective intra-aortic balloon pump or increasing volumes of contrast media). Increasing score numbers of $\leq 5,6$ to 10,11 to 16 , and $\geq 16$ conferred an increasing CI-AKI risk of $7.5 \%$, $14.0 \%, 26.1 \%$, and $57.3 \%$, respectively.

\section{Pathophysiology}

Several factors are related to the pathogenesis of CIAKI (Table 1), comprised of hemodynamic changes of renal blood flow which causes hypoxia in the renal medulla, direct toxic effect of the radiocontrast media on renal cells associated with renal endothelial 
dysfunction, and altered microcirculation (19,26-38). The administration of radiocontrast media leads to vasoconstriction, markedly affecting renal parenchymal oxygenation, especially in the outer medulla. After the injection of the high-osmolar ionic agent sodium iothalamate, cortical $\mathrm{PO}_{2}$ declined from 40 to $25 \mathrm{mmHg}$, and the already hypoxic outer medulla, $\mathrm{PO}_{2}$ fell from $26 \mathrm{mmHg}$ at baseline to mean levels as low as $9 \mathrm{mmHg}$ (39). A comparable decline of medullary $\mathrm{PO}_{2}$ from approximately 30 to $15 \mathrm{mmHg}$ with the administration of ionic and nonionic low-osmolar radiocontrast agents, as well as isoosmolar dyes has also been described (40). In addition, neurohumoral mediators such as nitric oxide, plasma endothelin, adenosine, prostaglandin and vasopressin likely contributed further to the changes in renal microcirculation. Mechanical factors involving viscosity also played a role in the complex system causing lowpressure medullary microcirculation. An increased plasma viscosity after radiocontrast media administration has been demonstrated to interfere with blood flow, particularly within the hypertonic environment of the (inner) renal medulla, where the plasma viscosity is already increased. Indeed, there are several animal studies that have shown a correlation between experimental CI-AKI and viscosity of the radiopaque compound $(35,36)$.

Evidence also suggested direct tubular toxicity on the proximal tubular cells from radiocontrast media, where there is an induced decline in tubule $\mathrm{K}^{+}$, adenosine triphosphate (ATP), and total adenine nucleotide contents, and an increase in $\mathrm{Ca}^{2+}$ content (38). These changes were more pronounced with the very high-osmolality ionic compound than with the lower osmolality nonionic compound. The cytotoxic effects were aggravated by hypoxia, indicating interactions between direct cellular mechanisms and vasoconstriction-mediated hypoxia $(36,37)$.

\section{Literature review}

Recent observational studies have raised questions on the prevalence and severity of CI-AKI following IV radiocontrast media, while other studies exploring the same subject have remained inconclusive due to the absence of control groups and adjustment with covariates associated with AKI. Indeed, prospective, randomized controlled trials published were performed to study the beneficial effects of different contrast materials used or prophylactic measures to prevent the development of CI-AKI, rather than addressing the question of the incidence of CI-AKI.
Furthermore, many interventions used to prevent CI-AKI may have had direct effects on the pathogenesis of AKI. For instance, the ability of volume expansion agents to decrease CI-AKI could be related to the hemodilution of serum creatinine level and correction of hypotension and viscosity in the medullary circulation. Many of the observational studies included data only from hospitalized patients, leading to selection bias toward a sicker population with multiple comorbid conditions associated with observed AKI independent of radiocontrast media exposure.

Numerous prospective and retrospective studies were unable to show increased rates of AKI in patients who received radiocontrast media $(14,15,41)$. A recent metaanalysis of 28 observational controlled studies involving 107,335 participants demonstrated that when compared with non-contrast CT, contrast-enhanced CT was not significantly associated with AKI [odds ratio (OR) 0.94; $95 \%$ confidence interval (CI): 0.83 to 1.07 (42)]. A systematic review and meta-analysis of controlled studies examining the incidence of AKI in patients exposed to radiocontrast media compared with patients who underwent an imaging examination without radiocontrast media or were otherwise unexposed (control group) of 13 nonrandomized studies representing 25,950 patients demonstrated a similar incidence of AKI, dialysis, and death between the contrast medium group and control group (43).

Wilhelm-Leen et al. sought to estimate the burden of AKI among 5.9 million hospitalizations among adult patients receiving radiocontrast, as compared to patients with similar comorbidity and severity of illness who did not receive radiocontrast during their hospitalization. Patients who received radiocontrast did not develop AKI at a clinically higher rate-the rates of development of AKI were $5.5 \%$ (radiocontrast administered) vs. 5.6\% (no radiocontrast exposure). After controlling for comorbidity and acuity of illness, radiocontrast media administration was associated with an OR for AKI of 0.93 [95\% CI, 0.88 to 0.97 (44)]. When adjusted for age, sex, mechanical ventilation, and comorbidity, the administration of radiocontrast was correlated with a $7.4 \%$ (95\% CI, $2.6 \%$ to $12.0 \%$ ) reduction in the odds of AKI. The study had several limitations including the inability to determine with certainty if the administration of contrast preceded the diagnosis of AKI within any hospitalization (since AKI was determined solely based on administrative data) which placed the validity of its results into question. The more generalizable implication of the study itself seemed to be that 'sicker' patients are more liable to develop an AKI, with 
or without the presence of contrast. A retrospective study with a total of 12,508 propensity score-matched patients reported that the incidence of AKI significantly increased with decreasing baseline eGFR $(\mathrm{P}<0.0001)$. However, this incidence was not significantly different between contrast material and non-contrast material groups in any eGFR subgroup, even in patients with eGFR of less than $30 \mathrm{~mL} / \mathrm{min} / 1.73 \mathrm{~m}^{2}(14)$.

Intra-arterial contrast exposure is reported to be more nephrotoxic than intravenous use. Several studies have shown higher rates of AKI after coronary angiography (13\%) compared to AKI rates with IV radiocontrast media exposure (5-6\%), potentially due to higher concentrations of radiocontrast media delivered to the kidney and the shorter transit time to the kidneys $(45,46)$. Patients undergoing primary PCI are at higher risk of CI-AKI compared with those undergoing elective interventions, though recently there has been data suggesting that risk of AKI with PCI may not be as pronounced as in prior studies. Caspi et al. used a prospective database to determine the causal association of contrast material exposure and the incidence of AKI following PCI using a control group of propensity score-matched patients with STEMI who were not exposed to contrast material (47). Among propensity score-matched patients, AKI rates were not significantly different with and without PCI $(8.6 \%$ versus $10.9 \%$, $\mathrm{P}=0.12)$. In the PCI cohort, independent predictors of AKI included age $\geq 70$ years, insulin-treated diabetes mellitus, diuretic therapy, anterior infarction, baseline estimated glomerular filtration rate, and variables related to the presence of pump failure (higher Killip class, intra-aortic balloon pump use) and reduced left ventricular ejection fraction but not contrast material dose. They concluded the risk for AKI was similar among STEMI patients with and without contrast material exposure. This study was an observational one where some patients were recruited during a period in which both PCI and thrombolysis were acceptable reperfusion strategies. Despite propensity score analysis, treatment bias related to age, baseline renal function, infarct location, and hemodynamic status may have affected the decision to perform PCI, resulting in heterogeneity caused by AKI-predisposing comorbidities between contrast and non-contrast groups. Nonetheless this study raised the question of whether CI-AKI truly exists in the setting of coronary angiography, and determined it is unlikely that radiocontrast media is a primary pathogenic factor responsible for renal dysfunction.

Brown et al. examined the associations between PCI, AKI and CKD progression to study long-term consequences of PCI and development/progression of CKD (48). They classified cardiac catheterization-associated AKI (CCAAKI) to represent a broad category of AKI that included contrast-induced nephropathy, as well as AKI attributable to atheroembolization, hypotension, and other causes associated with these procedures. A total of 48,810 patients were evaluated in three groups (PCI complicated by CCAAKI, uncomplicated PCI, and controls), with controls well matched to PCI patients for age, diabetes mellitus, hypertension, heart failure, and baseline CKD stage. Of note, CCA-AKI developed in $13 \%$ of the PCI cohort, which was consistent with other reports, and PCI patients developing CCA-AKI were more likely to have urgent or emergent indications for intervention, exhibited greater temporal proximity to the diagnostic cardiac catheterization, and had angiographic evidence of more severe coronary disease. While this study did overcome the limitations of some prior studies including a large sample size, using laboratory based eGFR values rather than administrative diagnosis codes, and using a control group to help validate their findings, it was limited primarily by the definition used for CCA-AKI (which essentially included any cause of AKI occuring following PCI), thus rendering the study unable to truly classify how much of a role CI-AKI had in the overall development of AKIs.

A subgroup analysis of the PRESERVE (Prevention of Serious Adverse Effects Following Angiography) trial assessed IV sodium bicarbonate or IV sodium chloride and oral acetylcysteine or placebo for the prevention of CI-AKI and intermediate-term adverse outcomes (49). A subgroup analysis was conducted of the efficacy of these interventions in patients who underwent PCI during the study angiographic examination. There were no significant between-group differences in the rates of CI-AKI. In conclusion, among patients with CKD undergoing PCI, there was no benefit of IV sodium bicarbonate over IV sodium chloride or of acetylcysteine over placebo for the prevention of CI-AKI or intermediate-term adverse outcomes. The study was limited by the reduced sample size from the overall PRESERVE trial, exclusion of patients CKD V or more acutely sick (STEMI), and the study population was predominantly male, impacting its generalizability. While this study did monitor several other factors that are likely to influence CI-AKI (such as volume and type of contrast used), it failed to consider other potential causes of AKI (such as peri-PCI hypotension), thus raising the question once again regarding the true incidence of CI-AKI. 
A recent study done examined data obtained from 323 consecutive patients with advanced renal dysfunction (eGFR $<30 \mathrm{~mL} / \mathrm{min} / 1.73 \mathrm{~m}^{2}$ ) to address the occurrence and prognostic influence of CIN in patients with advanced renal dysfunction (50). CI-AKI was observed in 31 patients $(9.7 \%)$, and a comparison between patients with and without CIN showed no significant intergroup differences in the occurrence of all-cause death or the initiation of permanent dialysis. The authors concluded that the incidence of CI-AKI was not higher in Japanese patients with advanced renal dysfunction in routine clinical practice, and that CIAKI did not show a significant prognostic influence.

\section{Summary and conclusions}

Contrast-induced AKI has become a topic of much debate in the practice of medicine. While a substantial amount of time and resources have been devoted to try and better define it or to disprove it, no study has so far succeeded in answering the question conclusively. In more recent years, there does seem to have been more of a trend of questioning the existence of CI-AKI as a true entity, with multiple studies failing to find any association between radiocontrast media exposure and renal injury. Several mechanisms have been proposed to explain the pathogenesis of CI-AKI, but it should be recalled that this data was derived from animal models. The diagnosis of CI-AKI, per its very definition, is based on the clinical presentation and time to development, and as such remains a diagnosis of exclusion. More recently, when AKI following contrast exposure cannot be ascribed to contrast exposure, the term post-contrast AKI has been labeled, emphasizing the temporal relationship between contrast exposure and AKI (19,51).

Several of the studies reviewed were comprehensive in their designs but neglected to account for other more commonly accepted causes of AKI that impacted the validity of their results. Other studies acknowledged other etiologies yet chose to group all AKIs under an umbrella term such as cardiac catheter-associated AKI, which did not help to clarify the incidence of CI-AKI (15). The occurrence of CI-AKI was likely overestimated in the past because of confounders for AKI. While none of the studies have managed to prove or disprove CI-AKI conclusively, they have built up several thought-provoking points regarding the subject. Patients with more severe illnesses are more predisposed to developing AKI independent of their risk of potential CI-AKI, and predictably, their overall prognosis is poor $(12,13,18)$. This raises concern for potentially life- saving testing/interventions being deferred or suspended for patients deemed at risk for AKI out of fear of causing CI-AKI. Preventative measures to counter potential CIAKI appear to be becoming the standard of care for several contrast-associated procedures, with questionable evidence as to whether the AKI that is being prevented is truly CI-AKI or another more common cause of AKI. Thus, labelling every AKI that follows a contrast study as CI-AKI without doing the due diligence of an appropriate workup would be considered a disservice to the patient.

The best way of settling the matter would be to design a randomized controlled trial of patients undergoing coronary angiography and/or PCI with and without CKD, while controlling for other risk factors and confounders. Each patient should have documented pre-procedure baseline renal function and urine studies (volume, sp. gravity, blood, protein levels), then have their perioperative vitals, cardiac status, blood loss recorded, followed by a postoperative tracking of daily serum creatinine for a minimum of 72 hours. The occurrence of an AKI should prompt an appropriate workup to rule out all other plausible etiologies before the determination of CI-AKI can be made. Since clinical assessment can be subjective, ideally it should be independently verified by two different physicians. Such a method may still not guarantee an absolute answer but should allow for a better determination of the incidence of CI-AKI than is available literature so far. Unfortunately, time, financial, and perhaps even ethical constraints would ensure that such an endeavor may be extremely challenging to arrange, and so a definitive answer on CI-AKI, and whether it really exists, may continue to elude clinicians.

\section{Acknowledgments}

Funding: None.

\section{Footnote}

Provenance and Peer Review: This article was commissioned by the Guest Editor (Ion S. Jovin) for the series "Interventional Cardiology" published in fournal of Thoracic Disease. The article was sent for external peer review organized by the Guest Editor and the editorial office.

Conflicts of Interest: The series "Interventional Cardiology" was commissioned by the editorial office without any funding or sponsorship. The authors have no other conflicts of interest to declare. 
Ethical Statement: The authors are accountable for all aspects of the work in ensuring that questions related to the accuracy or integrity of any part of the work are appropriately investigated and resolved.

Open Access Statement: This is an Open Access article distributed in accordance with the Creative Commons Attribution-NonCommercial-NoDerivs 4.0 International License (CC BY-NC-ND 4.0), which permits the noncommercial replication and distribution of the article with the strict proviso that no changes or edits are made and the original work is properly cited (including links to both the formal publication through the relevant DOI and the license). See: https://creativecommons.org/licenses/by-nc-nd/4.0/.

\section{References}

1. Bartels ED, Brun GC, Gammeltoft A, et al. Acute anuria following intravenous pyelography in a patient with myelomatosis. Acta Med Scand 1954;150:297-302.

2. Killmann SA, Gjorup S, Thaysen JH. Fatal acute renal failure following intravenous pyelography in a patient with multiple myeloma. Acta Med Scand 1957;158:43-6.

3. McCullough PA, Soman SS. Contrast-induced nephropathy. Crit Care Clin 2005;21:261-80.

4. Schilp J, de Blok C, Langelaan M, et al. Guideline adherence for identification and hydration of high-risk hospital patients for contrast-induced nephropathy. BMC Nephrol 2014;15:2 .

5. Nash K, Hafeez A, Hou S. Hospital-acquired renal insufficiency. Am J Kidney Dis 2002;39:930-6.

6. Lloyd-Jones D, Adams RJ, Brown TM, et al. Heart disease and stroke statistics--2010 update: a report from the American Heart Association. Circulation 2010;121:e46-215.

7. Rihal CS, Textor SC, Grill DE, et al. Incidence and prognostic importance of acute renal failure after percutaneous coronary intervention. Circulation 2002;105:2259-64.

8. Chong E, Shen L, Poh KK, et al. Risk scoring system for prediction of contrast-induced nephropathy in patients with pre-existing renal impairment undergoing percutaneous coronary intervention. Singapore Med J 2012;53:164-9.

9. Marenzi G, Lauri G, Assanelli E, et al. Contrast-induced nephropathy in patients undergoing primary angioplasty for acute myocardial infarction. J Am Coll Cardiol 2004;44:1780-5.

10. McCullough PA, Wolyn R, Rocher LL, et al. Acute renal failure after coronary intervention: incidence, risk factors, and relationship to mortality. Am J Med 1997;103:368-75.

11. Mohammed NM, Mahfouz A, Achkar K, et al. Contrast- induced Nephropathy. Heart Views 2013;14:106-16.

12. Feldkamp T, Kribben A. Contrast media induced nephropathy: definition, incidence, outcome, pathophysiology, risk factors and prevention. Minerva Med 2008;99:177-96.

13. McDonald RJ, McDonald JS, Carter RE, et al. Intravenous contrast material exposure is not an independent risk factor for dialysis or mortality. Radiology 2014;273:714-25.

14. McDonald JS, McDonald RJ, Carter RE, et al. Risk of intravenous contrast material-mediated acute kidney injury: a propensity score-matched study stratified by baseline-estimated glomerular filtration rate. Radiology 2014;271:65-73.

15. Newhouse JH, RoyChoudhury A. Quantitating contrast medium-induced nephropathy: controlling the controls. Radiology 2013;267:4-8.

16. Mehran R, Aymong ED, Nikolsky E, et al. A simple risk score for prediction of contrast-induced nephropathy after percutaneous coronary intervention: development and initial validation. J Am Coll Cardiol 2004;44:1393-9.

17. Bellomo R, Ronco C, Kellum JA, et al. Acute renal failure definition, outcome measures, animal models, fluid therapy and information technology needs: the Second International Consensus Conference of the Acute Dialysis Quality Initiative (ADQI) Group. Crit Care 2004;8:R204-12.

18. Section 4: Contrast-induced AKI. Kidney Int Suppl (2011) 2012;2:69-88.

19. Owen RJ, Hiremath S, Myers A, et al. Canadian Association of Radiologists consensus guidelines for the prevention of contrast-induced nephropathy: update 2012. Can Assoc Radiol J 2014;65:96-105.

20. McCullough PA. Contrast-induced acute kidney injury. J Am Coll Cardiol 2008;51:1419-28.

21. Gruberg L, Mintz GS, Mehran R, et al. The prognostic implications of further renal function deterioration within $48 \mathrm{~h}$ of interventional coronary procedures in patients with pre-existent chronic renal insufficiency. J Am Coll Cardiol 2000;36:1542-8.

22. Subramanian S, Tumlin J, Bapat B, et al. Economic burden of contrast-induced nephropathy: implications for prevention strategies. J Med Econ 2007;10:119-34.

23. Amin AP, Salisbury AC, McCullough PA, et al. Trends in the incidence of acute kidney injury in patients hospitalized with acute myocardial infarction. Arch Intern Med 2012;172:246-53.

24. McCullough PA, Choi JP, Feghali GA, et al. ContrastInduced Acute Kidney Injury. J Am Coll Cardiol 2016;68:1465-73.

25. Ozkok S, Ozkok A. Contrast-induced acute kidney 
injury: A review of practical points. World J Nephrol 2017;6:86-99.

26. Harjai KJ, Raizada A, Shenoy C, et al. A comparison of contemporary definitions of contrast nephropathy in patients undergoing percutaneous coronary intervention and a proposal for a novel nephropathy grading system. Am J Cardiol 2008;101:812-9.

27. Tumlin J, Stacul F, Adam A, et al. Pathophysiology of contrast-induced nephropathy. Am J Cardiol 2006;98:14K-20K.

28. Persson PB, Hansell P, Liss P. Pathophysiology of contrast medium-induced nephropathy. Kidney Int 2005;68:14-22.

29. Keaney JJ, Hannon CM, Murray PT. Contrast-induced acute kidney injury: how much contrast is safe? Nephrol Dial Transplant 2013;28:1376-83.

30. Seeliger E, Sendeski M, Rihal CS, et al. Contrast-induced kidney injury: mechanisms, risk factors, and prevention. Eur Heart J 2012;33:2007-15.

31. Katzberg RW, Barrett BJ. Risk of iodinated contrast material--induced nephropathy with intravenous administration. Radiology 2007;243:622-8.

32. Persson PB, Tepel M. Contrast medium-induced nephropathy: the pathophysiology. Kidney Int Suppl 2006:S8-10.

33. Liss P, Nygren A, Hansell P. Hypoperfusion in the renal outer medulla after injection of contrast media in rats. Acta Radiol 1999;40:521-7.

34. Nygren A, Ulfendahl HR. Effects of high- and lowosmolar contrast media on renal plasma flow and glomerular filtration rate in euvolaemic and dehydrated rats. A comparison between ioxithalamate, iopamidol, iohexol and ioxaglate. Acta Radiol 1989;30:383-9.

35. Nygren A, Ulfendahl HR, Hansell P, et al. Effects of intravenous contrast media on cortical and medullary blood flow in the rat kidney. Invest Radiol 1988;23:753-61.

36. Ueda J, Nygren A, Sjoquist M, et al. Iodine concentrations in the rat kidney measured by X-ray microanalysis. Comparison of concentrations and viscosities in the proximal tubules and renal pelvis after intravenous injections of contrast media. Acta Radiol 1998;39:90-5.

37. Lancelot E, Idee JM, Couturier V, et al. Influence of the viscosity of iodixanol on medullary and cortical blood flow in the rat kidney: a potential cause of Nephrotoxicity. J Appl Toxicol 1999;19:341-6.

38. Humes HD, Hunt DA, White MD. Direct toxic effect of the radiocontrast agent diatrizoate on renal proximal tubule cells. Am J Physiol 1987;252:F246-55.

39. Heyman SN, Brezis M, Epstein FH, et al. Early renal medullary hypoxic injury from radiocontrast and indomethacin. Kidney Int 1991;40:632-42.
40. Liss P, Nygren A, Erikson U, et al. Injection of low and iso-osmolar contrast medium decreases oxygen tension in the renal medulla. Kidney Int 1998;53:698-702.

41. Bruce RJ, Djamali A, Shinki K, et al. Background fluctuation of kidney function versus contrast-induced nephrotoxicity. AJR Am J Roentgenol 2009;192:711-8.

42. Aycock RD, Westafer LM, Boxen JL, et al. Acute Kidney Injury After Computed Tomography: A Meta-analysis. Ann Emerg Med 2018;71:44-53.e4.

43. McDonald JS, McDonald RJ, Comin J, et al. Frequency of acute kidney injury following intravenous contrast medium administration: a systematic review and meta-analysis. Radiology 2013;267:119-28.

44. Wilhelm-Leen E, Montez-Rath ME, Chertow G. Estimating the Risk of Radiocontrast-Associated Nephropathy. J Am Soc Nephrol 2017;28:653-9.

45. Solomon R. Contrast-induced acute kidney injury: is there a risk after intravenous contrast? Clin J Am Soc Nephrol 2008;3:1242-3.

46. Dong M, Jiao Z, Liu T, et al. Effect of administration route on the renal safety of contrast agents: a meta-analysis of randomized controlled trials. J Nephrol 2012;25:290-301.

47. Caspi O, Habib M, Cohen Y, et al. Acute Kidney Injury After Primary Angioplasty: Is Contrast-Induced Nephropathy the Culprit? J Am Heart Assoc 2017. doi: 10.1161/JAHA.117.005715.

48. Brown JR, Solomon RJ, Robey RB, et al. Chronic Kidney Disease Progression and Cardiovascular Outcomes Following Cardiac Catheterization-A Population-Controlled Study. J Am Heart Assoc 2016. doi: 10.1161/JAHA.116.003812.

49. Garcia S, Bhatt DL, Gallagher M, et al. Strategies to Reduce Acute Kidney Injury and Improve Clinical Outcomes Following Percutaneous Coronary Intervention: A Subgroup Analysis of the PRESERVE Trial. JACC Cardiovasc Interv 2018;11:2254-61.

50. Negishi Y, Tanaka A, Ishii H, et al. Contrast-Induced Nephropathy and Long-Term Clinical Outcomes Following Percutaneous Coronary Intervention in Patients With Advanced Renal Dysfunction (Estimated Glomerular Filtration Rate $<30$ ml/min/1.73 m(2)). Am J Cardiol 2019;123:361-7.

51. Vandenberghe W, Hoste E. Contrast-associated acute kidney injury: does it really exist, and if so, what to do about it? F1000Res 2019 doi: 10.12688/ f1000research.16347.1.

Cite this article as: Haq MFU, Yip CS, Arora P. The conundrum of contrast-induced acute kidney injury. J Thorac Dis 2020;12(4):1721-1727. doi: 10.21037/jtd.2019.12.88 